
\section{Coluste}

${ }^{1}$ Department of Internal Medicine, Beth Israel Deaconess Medical Center, Harvard Medical School, Boston, Massachusetts, USA

${ }^{2}$ Department of Medicine, Brigham and Women's Hospital, Boston, Massachusetts, USA ${ }^{3}$ Susan F Smith Center for Women's Cancer, Dana Farber Cancer Institute, Harvard Medical School, Boston, Massachusetts, USA

${ }^{4}$ Department of Pulmonary and Critical Care Medicine, The Ohio State University, Columbus, Ohio, USA

\section{Correspondence to}

Dr Chelsea Ma, Department of Internal Medicine, Beth Israel Deaconess Medical Center, Harvard Medical School, 330 Brookline Ave, Boston, MA 02215, USA; chcma@ucdavis. edu

Received 21 August 2016 Revised 1 May 2017 Accepted 9 May 2017

\section{CrossMark}

To cite: Ma C, Frosch ZAK, Overmoyer B, et al. Thorax 2018;73:97-98.

\title{
Vocal fold paralysis on positron emission tomography-CT
}

\author{
Chelsea Ma, ${ }^{1}$ Zachary AK Frosch, ${ }^{2}$ Beth Overmoyer, ${ }^{3}$ Avraham Z Cooper ${ }^{4}$
}

\section{CASE}

A 58-year-old woman with a 40 pack-year smoking history presented with several weeks of shortness of breath, chest pain radiating to the back and a hoarse voice. Initial chest X-ray showed a new left upper lobe mass concerning for malignancy. She then underwent positron emission tomography(PET)-CT, which revealed a fluorodeoxyglucose (FDG)-avid left hilar lung mass, (figure 1, red arrow) and left supraclavicular and mediastinal lymphadenopathy (figure 1, orange and green arrows). The PET-CT also showed asymmetric FDG uptake of the vocal folds at the level of the thyroid cartilage (right greater than left), suggestive of left vocal fold paralysis (figure 2, yellow arrow). A CT image of the vocal folds is depicted in figure 3.

Such asymmetric FDG uptake as seen in this patient is thought to result from decreased and increased glucose consumption by the paralysed and functioning vocal folds, respectively. Asymmetry in FDG uptake is more prominent when paralysis is acute or subacute rather than chronic. ${ }^{1}$ The most likely aetiology of vocal fold paralysis in this patient was compression of the left recurrent laryngeal nerve by either the hilar lung mass or the associated mediastinal lymphadenopathy. This nerve courses below the aortic arch posterior to the ligamentum arteriosum, which appears to correspond to the area where the hilar mass and mediastinal lymphadenopathy were located. ${ }^{2}$ It is more common to see left rather than right recurrent laryngeal nerve compression due to its longer course and passage through the aortopulmonary window. Such compression would explain the patient's complaint of hoarseness, which is commonly the earliest sign of compression or invasion of the recurrent laryngeal nerve.

The patient underwent endoscopic biopsy, which revealed small cell lung carcinoma. Further invasive
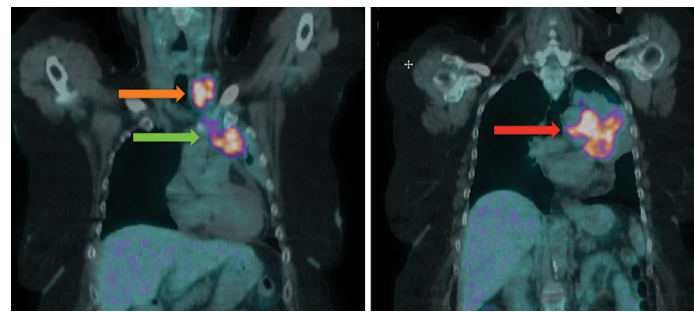

Figure 1 PET-CT of the chest showing FDG-avid left hilar mass (red arrow) and left supraclavicular and mediastinal lymphadenopathy (orange and green arrows). FDG, fluorodeoxyglucose; PET, positron emission tomography.

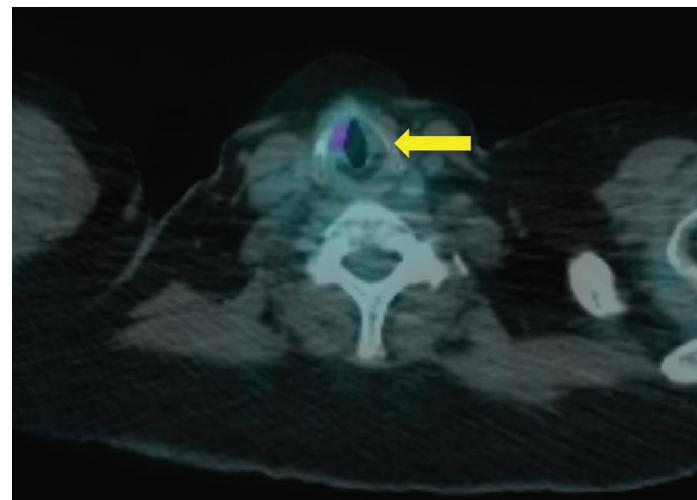

Figure 2 PET-CT showing asymmetric FDG uptake of vocal folds at the level of the thyroid cartilage (yellow arrow). FDG, fluorodeoxyglucose; PET, positron emission tomography.

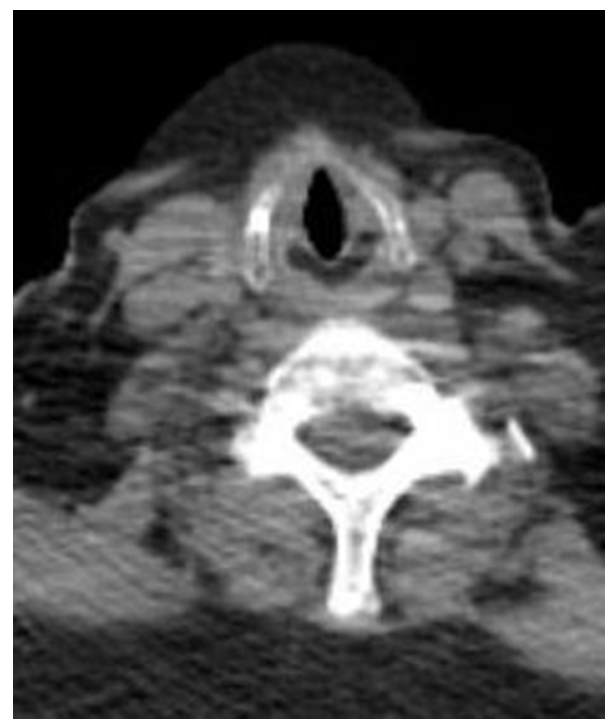

Figure 3 CT of the vocal folds at the level of the thyroid cartilage.

assessment to evaluate hoarseness was not needed, as the PET-CT was diagnostic. She was determined to have limited stage small cell lung carcinoma, and was started on cisplatin and etoposide as an inpatient to minimise symptoms and reduce disease burden. Chemotherapy plus radiation therapy were continued as an outpatient. After about 1 month of therapy, the patient reported improvement in both dyspnoea and hoarseness.

Contributors Drs CM, ZAKF, BO and AZC all contributed to the planning, conduct and reporting of the manuscript. $\mathrm{Dr} \mathrm{CM}$ is responsible for the overall content as guarantor. 


\section{Competing interests None declared.}

\section{Patient consent Obtained}

Provenance and peer review Not commissioned; externally peer reviewed.

(c) Article author(s) (or their employer(s) unless otherwise stated in the text of the article) 2018. All rights reserved. No commercial use is permitted unless otherwise expressly granted.

\section{REFERENCES}

1 Komissarova M, Wong KK, Piert M, et al. Spectrum of 18F-FDG PET/CT findings in oncology-related recurrent laryngeal nerve palsy. AJR Am J Roentgenol 2009; 192:288-94

2 Paquette CM, Manos DC, Psooy BJ. Unilateral vocal cord paralysis: a review of CT findings, mediastinal causes, and the course of the recurrent laryngeal nerves. Radiographics 2012;32:721-40. 